\title{
Etnia mapuche y vulnerabilidad: una mirada desde los indicadores de carencialidad socioeducativa
}

\author{
Carlos Rodríguez Garcés \\ Doctor por la Universidad de Barcelona \\ Director del Centro de Investigación CIDCIE \\ Universidad de Bío-Bío \\ carlosro@ubiobio.cl \\ Casilla 447, Chillán, Chile. \\ https://orcid.org/0000-0002-9346-0780
}

\section{Geraldo Padilla Fuentes \\ Licenciado y Trabajado Social \\ Investigador adscrito al Centro de Investigación CIDCIE \\ Universidad de Bío-Bío \\ gpadilla@ubiobio.cl \\ Casilla 447, Chillán, Chile. \\ https://orcid.org/0000-0003-0882-1818}

Claudia Suazo Ruiz

Alumna Carrera Trabajo Social Universidad del Bío-Bío

Ayudante del Centro de Investigación CIDCIE

Universidad de Bío-Bío

claudia.suazo1601@alumnos.ubiobio.cl

https://orcid.org/0000-0002-9937-4875

\begin{abstract}
How to cite this paper:
Rodríguez-Garcés, Carlos; Padilla-Fuentes, Geraldo; Suazo-Ruiz, Claudia (2020). Etnia mapuche y vulnerabilidad: una mirada desde los indicadores de carencialidad socioeducativa. Revista Encuentros, Universidad Autónoma del Caribe. Vol. 18-01.
\end{abstract}

Doi: $10.15665 /$ encuent.v18i01.2232.

Recibido: 18 de diciembre de 2019 / Aceptado: 16 de diciembre de 2019

\section{RESUMEN}

Las comunidades mapuches han enfrentado una persiste exclusión territorial y simbólica en los servicios educativos que el Estado ha dispuesto para su población nacional. Por lo anterior, este artículo analiza la situación de vulnerabilidad socioeducativa que afecta a la población con ascendencia mapuche residente en la macrozona sur desde una perspectiva multidimensional; el diseño es cuantitativo, descriptivo y transversal. Los resultados muestran que la población mapuche cuenta con mayores niveles de ruralidad y pobreza. Entre los indicadores de carencia multidimensional, Escolaridad y Entorno registran la mayor prevalencia. En cuanto a calidad educativa, los y las estudiantes mapuches alcanzan menores puntajes en test estandarizados SIMCE y asisten mayoritariamente a establecimientos educativos municipales y que atienden a población vulnerable. Así también, comparten salón de clase con estudiantes de bajo rendimiento y dominio curricular. Las conclusiones resaltan la necesidad de atender las diferencias culturales y brechas educativas existentes para generar un sistema educativo más inclusivo con la población indígena y que brinde iguales oportunidades de acceso, permanencia y calidad.

Palabras clave: Rendimiento escolar, segregación escolar, Pobreza multidimensional, etnia Mapuche, conflictos culturales. 


\title{
Mapuche ethnicity and vulnerability: a look from the indicators of socioeducational deficiency
}

\begin{abstract}
Mapuche communities have faced a persistent territorial and symbolic exclusion in the educational services that the State has provided for its national population. Therefore, this article analyzes the situation of socio-educational vulnerability that affects the population with Mapuche ancestry residing in the southern macrozone from a multidimensional perspective. The design is quantitative, descriptive and transversal. The results show that the Mapuche population has higher levels of rurality and poverty. Among the indicators of multidimensional poverty, Schooling and Environment showed the highest prevalence. In terms of educational quality, Mapuche students achieve lower scores on standardized SIMCE tests and mostly attend municipal educational establishments that serve vulnerable populations. Moreover, they share classroom with low-performing students and curricular proficiency. The conclusions indicate the need to resolve existing cultural differences and educational gaps to generate a more inclusive educational system with the indigenous population and that provides equal opportunities for access, permanence and quality.
\end{abstract}

Key words: Academic achievement, school segregation, multidimensional poverty, ethnicity, cultural minorities.

\section{Etnia mapuche e vulnerabilidade: um olhar a partir dos indicadores de deficiência socioeducacional}

\begin{abstract}
RESUMO
As comunidades mapuche enfrentam uma exclusão territorial e simbólica persistente nos serviços educacionais que o Estado oferece à sua população nacional. Portanto, este artigo analisa a situação de vulnerabilidade socioeducativa que afeta a população com ascendência mapuche que reside na macrozona sul sob uma perspectiva multidimensional; o desenho é quantitativo, descritivo e transversal. Os resultados mostram que a população mapuche tem níveis mais altos de ruralidade e pobreza. Dentre os indicadores de falta multidimensional, Escolaridade e Meio Ambiente registraram a maior prevalência. Em termos de qualidade educacional, os estudantes Mapuche alcançam notas mais baixas nos testes SIMCE padronizados e freqüentam principalmente estabelecimentos de ensino municipais que atendem populações vulneráveis. Da mesma forma, eles compartilham uma sala de aula com alunos de baixo desempenho e proficiência curricular. As conclusões destacam a necessidade de abordar as diferenças culturais existentes e as lacunas educacionais para gerar um sistema educacional mais inclusivo com a população indígena e que ofereça oportunidades iguais de acesso, permanência e qualidade.
\end{abstract}

Palavras chave: Desempenho escolar, segregação escolar, pobreza multidimensional, etnia mapuche, conflitos culturais.

\section{Introducción}

Las naciones han relevado la importancia de la heterogeneidad étnica y cultural como atributo distintivo y enriquecedor de las sociedades modernas, particularmente importante en contextos de globalización e internacionalización (Henry, 2010). En lo que al Estado chileno respecta, la relación establecida con los pueblos originarios ha sido históricamente tensa y problemática, manteniendo en la actualidad un conflicto abierto en lo que refiere a la pauperización de sus derechos territoriales y calidad de vida. En efecto, la población indígena convive en situaciones de pobreza, marginación y rechazo como expresión de un racismo interiorizado en la sociedad mayor (Segato, 2012).

El histórico desamparo del que han sido objeto pueblos originarios como el Mapuche es el resultado de una serie de determinantes sociales y culturales que, en su especificidad contextual y dinámica, han profundizado 
su vulnerabilidad en términos objetivos y perceptivos. Los bajos ingresos, el desempleo, la discriminación de género y los bajos niveles de escolaridad a los que han estado expuestos son expresión de ello (Montero y Garcés, 2009; Juárez-Ramírez, et al., 2014).

Los mapuches han sido concebidos como un grupo rural, de tradición agrícola y economías comunitarias (Cunningham, 2010). Atributos que si bien persisten, han mutado significativamente. El empobrecimiento y reducción predial han afectado la producción agrícola, lo que unido a la competencia de mercado y su incidencia en los precios ponen en riesgo la sustentabilidad económica y fuerzan a la migración, principalmente de la población más joven. A estos eventos se añaden los costos de un modelo de desarrollo depredador del medio ambiente, el cual mediante actividades mineras, proyectos hidroeléctricos e industria salmonera y forestal, pone en riesgo la vida y salud de los pueblos originarios, empobrece el territorio y fuerza su desplazamiento (Bolados, 2012).

Como resultado del nivel de exposición a riesgos, los bajos activos económicos del hogar y la debilidad de los mecanismos de protección social, se acentúa la condición de pobreza y vulnerabilidad, particularmente en el pueblo mapuche (Becerra, Beldaño, Dastro y Coñuepan, 2011; Raguileo y Escobar, 2016). En este contexto, el campo educativo ha sido una matriz compartida de reproducción de desigualdades en lo que refiere a provisión y calidad del servicio educativo, capacidad para instalar valor agregado y generar oportunidades de movilidad social. No solo tienen una mayor probabilidad de ser pobres, sino también un menor acceso a la educación y probabilidad de culminar estudios, lo que se traduce en una menor acumulación de capital educativo, menores ingresos y estabilidad laboral (Trivelli, 2008; Vejar, Marchant y Peña, 2018).

El sistema educativo nacional exhibe altos niveles de segregación, lo que unido a la discriminación étnica y cultural hace de la escuela un espacio escasamente inclusivo. En ella se desarrollarían operaciones recurrentes de separación y jerarquización, donde lo étnico es invisibilizado, cuando no negado, a través de mecanismos simbólicos y acciones cotidianas (Hopenhayn y Bello, 2001). Discriminación y segregación que está a la base de las brechas entre población indígena y no-indígena respecto de analfabetismo, años de escolaridad, abandono escolar, continuación de estudios y rendimiento escolar.

En efecto, la población indígena no tan solo exhibe los menores índices educativos, sino que además la desigualdad se profundiza en contextos educativos de alta composición étnica (Mickelson, Bottia y Lambert, 2013; Webb, Canales y Becerra, 2017). Déficits educativos que no son resultado exclusivo de sus desventajas, asociadas a condiciones socioeconómicas y culturales familiares y del entorno escolar, sino también de factores personales generalmente no observados, tales como la motivación y la autoestima (Sanchez, 2011).

Si bien ha habido esfuerzos para promover e inculcar a las nuevas generaciones en la convivencia, respeto y tolerancia a la diversidad cultural dentro del aula (TurraDíaz, 2012; Miller, 2012; Quintrileo, Yáñez y Valenzuela, 2013; Serrano, Ponce de León, 2018), el sistema educativo nacional promueve un individualismo y competencia que distancia de un ethos cultural fundamentado en la cooperación y vida en comunidad (Arias y Bravo, 2019). En este contexto, los y las estudiantes mapuches enfrentan problemas para relacionarse y afianzar vínculos en un ambiente de monoculturalidad que les resulta identitariamente ajeno, lo que asociado a los limitados recursos familiares, expectativas parentales y proyecciones personales, obstaculizan la inserción y continuación de estudios (Quintriqueo, Torres, 2012; Quintrileo, Yáñez y Valenzuela, 2013; Espinoza, Castillo, González y Loyola, 2014; Fernández y Fuentes, 2018).

En un sistema educativo que privilegia características de estatus, clase y etnia, los y las estudiantes mapuches se enfrentan a una doble vulnerabilidad. Por una parte, deben integrarse a un currículum centralizado y monocultural donde el respeto a los valores y saberes indígenas es retórico y anecdótico; por otro lado, deben luchar por descontinuar históricas brechas de acumulación de capital humano y cultural, donde usualmente sus familias no cuentan con los recursos necesarios para apoyar las actividades escolares o formar parte de una comunidad educativa de la cual no se sienten parte. Este escenario de segregación y exclusión vulnera el pacto que la escuela establece con las familias en tanto entidad promotora de la movilidad social e instalación de las habilidades necesarias para desenvolverse (Vain, 2009). 
Así entendido, resulta cuestionable que en un espacio social cada vez más diverso y multicultural, la escuela se constituya en un mecanismo de reproducción de desigualdad social, particularmente para pueblos indígenas. Por tal motivo, resulta de interés analizar la situación de vulnerabilidad socioeconómica entre población mapuche, específicamente en lo que refiere a la dimensión educativa dentro de un paradigma de pobreza multidimensional. Se exploran tendencias y prevalencias de los indicadores de carencialidad y aprovechamiento educativo a nivel de hogares y unidades educativas que acogen a población indígena, utilizando bases de datos oficiales para la macrozona sur en Chile.

\section{Metodología}

\section{Diseño}

Se aplicó un diseño cuantitativo analítico, de corte transversal y carácter descriptivo. Se utilizan tablas de análisis bivariado con cálculos de media y proporción, los cuales abordan, en primer lugar, la situación de vulnerabilidad socioeconómica de población con pertenencia indígena residente en la macro zona sur, con énfasis en la perspectiva multidimensional de medición de la pobreza y su componente educativo. En segundo lugar, los datos exponen la situación socioeducativa de los Niños, Niñas y adolescentes (NNA) mapuche que participaron de los procesos SIMCE 2018, analizando sus niveles de logro en Lenguaje y Matemáticas, características de la unidad educativa a la que asisten y atributos escolares del grupo familiar.

\section{Instrumento}

El procesamiento de datos incluyó dos fuentes de información. La primera corresponde a la Encuesta de Caracterización Socioeconómica Nacional (CASEN) del año 2017, la cual permite caracterizar en términos territoriales, socioeconómicos y educativos a la población. CASEN es un instrumento que sirve para el diseño y evaluación de políticas sociales y focalización de recursos entre hogares. La encuesta CASEN está estructurada en 8 módulos que abordan las distintas dimensiones a nivel de persona, hogary vivienda. Para efectos de esta investigación, se utilizan particularmente ítems pertenecientes a Registro de residentes (Módulo H), Educación (Módulo E) e Identidades, redes y participación (Módulo $\mathrm{R}$ ).
La segunda fuente de información son los resultados del proceso de medición SIMCE 2018, entregados por la Agencia de Calidad de la Educación, en particular los puntajes obtenidos en test estandarizados y atributos reportados en los Cuestionarios de Calidad y Contexto de la Educación.

\section{Población y muestra}

El levantamiento de datos utilizado por CASEN corresponde a muestreos estratificados trietápicos. La encuesta tiene una representación nacional, con indicadores de error absoluto de 0,4 puntos porcentuales y $4,3 \%$ como error relativo, en un nivel de confianza al $95 \%$.

Por su parte, las bases de datos SIMCE incluyen información de todos los y las estudiantes que asisten a establecimientos educativos subvencionados por el Estado, sea en su calidad de Municipales o Particulares. Los datos abarcan el territorio nacional y la muestra se enfoca en aquellos ubicados en la macrozona sur.

\section{Procedimiento}

El procesamiento y análisis de la información transcurre por tres etapas recursivas. Se comienza por describir la proporción de población nacional con pertenencia indígena, región de residencia y situación de pobreza, tanto desde la perspectiva multidimensional como por ingresos.

Luego, se exponen los indicadores de pobreza multidimensional entre personas con pertenencia étnica de la macrozona sur. Se compara entre grupos familiares con y sin ascendencia indígena, reportando niveles de carencialidad educativa, de entorno y accesibilidad a servicios sociales.

Por último, se caracteriza la situación educativa de NNA mapuche y no-mapuche en términos de logro SIMCE, capital educativo parental y atributos socioeconómicos y de administración del establecimiento al que asisten.

\section{Análisis y resultados}

La diferenciación cultural, política y social que enfrenta la población con pertenencia a grupos étnicos, si bien se ha reducido con el paso de los años, continúa abarcando múltiples dimensiones de su vida comunitaria. El 
aislamiento que históricamente han debido enfrentar los grupos indígenas tiene huellas no tan solo simbólicas, sino también tangibles y localizadas en los territorios que habitan. Sirva de ejemplo su marcada residencia rural, la que, si bien no indica por sí misma vulnerabilidad, al sumarse con problemas de conectividad, pobreza y disponibilidad/calidad de servicios (médicos, educativos, sociales) se vuelve una fuerte condicionante para el bienestar. Obstáculos que, al igual que la densidad indígena, varían en razón de las características regionales.

Tabla 1: Población indígena según zona

\begin{tabular}{lccc}
\hline & Rural & Urbana & General \\
\hline $\begin{array}{l}\text { Población general } \\
\text { - Indígena }\end{array}$ & 18,5 & 8,2 & 12,7 \\
\hline $\begin{array}{l}\text { Población indígena macrozona sur } \\
\text { - Bío-Bío y Nuble }\end{array}$ & 7,0 & 23,8 & 16,2 \\
- LaAraucanía & 58,7 & 29,7 & 42,8 \\
- Los Ríosy Los Lagos & 34,3 & 46,5 & 40,9 \\
\hline $\begin{array}{l}\text { Pobreza indígena por macrozona sur } \\
\text { - Poringresos }\end{array}$ & 28,1 & 14,4 & 20,6 \\
- Multidimensional & 57,4 & 22,3 & 38,0 \\
\hline
\end{tabular}

Elaboración propia con datos CASEN, 2017.

A este respecto, CASEN informa que al año 2017 un $12,7 \%$ de la población chilena se identifica con algún grupo indígena, de los cuales un $45,6 \%$ es habitante de la macrozona sur, territorio donde un $45,2 \%$ habita sectores rurales (Tabla 1). Las regiones con mayor presencia de personas vinculadas a comunidades indígenas en la macrozona sur corresponden a la Araucanía (42,8\%), Los Rios y Los Lagos (40,9\%) y Bío-Bío y Nuble (16,2\%).

Usualmente, las características geográficasyeconómicas de estos territorios presionan hacia la migración urbana de las personas en busca de mejores oportunidades educativas, laborales y de salud. Comparativamente, el índice de ruralidad es mayor entre población indígena que no-indígena (,38 vs ,21), no obstante, el proceso de desruralización continúa.

Prescindir de la movilidad espacial expone a situaciones de carencia, acentuadas por características como el nivel educativo, tipo de empleo y capital económico de las personas. Tal es la relevancia que ha alcanzado el fenómeno migratorio campo-ciudad, particularmente entre población joven y de mayor nivel educativo, que la identidad étnica ya no se limita a la ruralidad. En la macrozona sur, un $54,8 \%$ de las personas que declara pertenencia indígena residen en zonas urbanas, cifra que incrementa al considerar jóvenes entre 15 y 25 años $(59,4 \%)$.

Aparejado a esta localización rural, encontramos que las situaciones de pobreza, sea desde una medición por ingresos (total percápita mensual) o multidimensional (carencias), tienden a la prevalencia entre quienes declaran identificación indígena. Siguiendo los datos aportados por CASEN, en estos grupos familiares hay un 20,6\% de personas en condición de pobreza por ingresos, mientras que desde el enfoque de carencias la cifra sube hasta un $38,0 \%$. Con independencia del enfoque, las estadísticas oficiales en Chile informan que la pobreza es más acentuada en zonas rurales, particularmente cuando de población indígena se trata.

Las diferencias de vulnerabilidad social entre grupos indígenas y no-indígenas están retratadas en múltiples dimensiones de la vida social, entre las cuales destacan representación política, cultura, trabajo y educación (Rapimán, 2005; Espinoza, 2018), las cuales son pesquisadas por el enfoque multidimensional de la pobreza (Tabla 2).

\begin{tabular}{|c|c|c|}
\hline & No etnia & Etnia \\
\hline Asistencia & 2,2 & 3,6 \\
\hline Rezago escolar & 2,5 & 4,0 \\
\hline Escolaridad & 35,0 & 52,3 \\
\hline Entorno & 8,4 & 16,5 \\
\hline Accesibilidad & 6,1 & 14,3 \\
\hline
\end{tabular}

Desde esta perspectiva, la carencia más acentuada entre población indígena corresponde a Escolaridad. Un $52,3 \%$ de estos hogares registra la presencia de al menos un miembro con nivel educativo inferior a la enseñanza mínima obligatoria correspondiente. Si bien se observa una creciente tendencia a la acumulación de capital educativo, particularmente por los más jóvenes, los niveles de escolaridad han sido históricamente bajos entre pueblos originarios. Situación que la literatura vincula con la segregación territorial y discriminación étnicacultural presentes en el sistema educativo formal y que obstaculizan su permanencia y egreso, presionando hacia la deserción escolar temprana (Cunningham, 2010). 
En los últimos 20 años, el sistema educativo nacional ha aumentado significativamente sus tasas de cobertura y promoción, situación que se expresa en los bajos índices de las dimensiones Asistencia y Rezago escolar, no obstante, se registran diferencias significativas entre población indígena y no indígena. En consistencia con la evidencia disponible, los datos informan de las dificultades que la población indígena tiene para dar continuidad a sus trayectorias educativas, en especial en territorios donde la oferta es escasa o inexistente (Gallart y Henríquez, 2006; UNESCO, 2009). Aunque esto implica incrementar los costos de desplazamiento y alejarse temporalmente de la familia (Mendoza, 2017), se evidencia un creciente interés por parte de la población en edad escolar por asistir a algún establecimiento educacional para dar continuidad a su trayectoria escolar.

A nivel de población indígena se observan acotados niveles de Rezago escolar. Pese a contrastar con el 2,5\% de la población no indígena, un escaso $4 \%$ de ellos reside en hogares donde alguno de los integrantes en edad escolar presenta un retraso educativo igual o superior a dos años lectivos. La política educativa ha realizado un esfuerzo por flexibilizar y adaptar los planes curriculares de manera de hacer más significativos los aprendizajes y mejorar las tasas de eficiencia interna del sistema, lo que ha impactado en los niveles de retención y promoción; empero, los problemas de calidad, segmentación e inclusión intercultural persisten (Webb, Canales y Becerra, 2017).

Si bien los territorios han mejorado notoriamente sus niveles de conectividad y dotación de infraestructura social y comunitaria, las carencias continúan, particularmente en lo que a población indígena refiere. Sus índices de vulnerabilidad en las dimensiones de Entorno y Accesibilidad son elevados. Un 16,5\% de la población indígena vive en hogares que registran problemas de contaminación medioambiental en su área residencial, mientras un $14,3 \%$ no dispone de equipamientos básicos o infraestructura comunitaria a una distancia cercana a la vivienda, tales como colegios, transporte público y centros de salud. Prevalencias que contrastan significativamente con las encontradas entre población no-indígena (Tabla 2).

Estas privaciones acentúan la pobreza monetaria y el sentimiento de marginalidad, condicionando la migración temporal o permanente, particularmente de los jóvenes que buscan mejores oportunidades laborales y educativas (Noe, Rodríguez y Zuñiga, 2005; Correa, 2018).

Las carencias en la dimensión educativa de los hogares indígena, evidenciadas por el enfoque multidimensional de la pobreza, encuentran su correlato en los indicadores de aprovechamiento educativo que alcanzan los estudiantes mapuches. Como expone la Tabla 3, sus puntajes en las pruebas estandarizadas SIMCE son significativamente menores que los registrados por estudiantes no mapuches, tanto en Lenguaje $[t=-15,267$; $\left.p<0.000\left(I_{95}=-9,3--7,2\right)\right]$ como en Matemáticas $[t=-$ 25,801; $\left.p<0.000\left(I_{95}={ }^{-19,0}-{ }^{-16,3}\right)\right]$, con diferencias particularmente elevadas en esta última.

No solo los puntajes exhibidos en pruebas estandarizadas son bajos, sino que también lo son las del resto de sus compañeros de aula. Un 25,7\% de los niños/as mapuches estudia en establecimientos donde al menos 3 de cada 4 estudiantes se encuentra en estándar de aprendizaje Insuficiente, es decir, no comprende ni aplica los contenidos y procedimientos básicos de Matemáticas. Si bien para Lenguaje la proporción es menor $(15,8 \%)$, las brechas son igualmente significativas $[\mathrm{p}<0,00]$.

Tabla 3: Caracterización socioeducativa según pertenencia étnica en macrozona sur

\begin{tabular}{|c|c|c|}
\hline & Mapuche & No mapuche \\
\hline $\begin{array}{l}\text { Nivel de logro SIMCE }[x(d s)] \\
\text { - Pje Matemáticas } \\
\text { - Pje Lenguaje }\end{array}$ & $\begin{array}{l}250,1(58,5) \\
245,2(46,4)\end{array}$ & $\begin{array}{l}267,8(63,7) \\
253,4(48,4)\end{array}$ \\
\hline $\begin{array}{l}\text { Alta insuficiencia SIMCE }(\%) \\
\text { - Matemáticas } \\
\text { - Lenguaje }\end{array}$ & $\begin{array}{l}25,7 \\
15,8\end{array}$ & $\begin{array}{l}15,4 \\
10,1\end{array}$ \\
\hline $\begin{array}{l}\text { Capital educativo parental }[x(d s)] \\
\text { - Padre } \\
\text { - Madre }\end{array}$ & $\begin{array}{l}10,2(3,4) \\
10,4(3,5)\end{array}$ & $\begin{array}{l}11,7(3,7) \\
11,8(3,5)\end{array}$ \\
\hline $\begin{array}{l}\text { GSE }(\%) \\
\text { - Bajo y Medio bajo } \\
\text { - Medio } \\
\text { - Medio alto y Alto }\end{array}$ & $\begin{array}{l}83,7 \\
12,5 \\
3,7\end{array}$ & $\begin{array}{l}66,8 \\
19,6 \\
13,6\end{array}$ \\
\hline Dependencia Municipal (\%) & 49,7 & 40,6 \\
\hline
\end{tabular}

Los bajos niveles de logro han sido una constante estructural del sistema educativo nacional, segmentados con base a la disponibilidad de recursos económicos y profundizados en razón de características como la pertenencia étnica y capacidad de la escuela para nivelar 
los diferenciados capitales culturales (Moreno-Doña, Gamboa, 2014; Fernández y Hauri, 2016; Ortega, 2019). Mecanismos como la libre elección educativa y selección escolar agravan la segmentación y guetificación del sistema escolar chileno, por lo que tiende a ser habitual que el alumnado de una misma escuela comparta perfil académico y socioeconómico, a la vez que se maximizan las diferencias entre comunidades escolares (Peña, 2007). Atributo que si bien es transversal, se acentúa en contextos de pertenencia étnica.

A objeto de perfilar la demanda, las instituciones educativas implementan mecanismos de selección basados en criterios escasamente transparentes y objetivos, lo que da pie a discriminar por condición socioeconómica o étnica. Por otra parte, padres y madres en el contexto de libre elección instalado prefieren que sus hijos/as interactúen con sujetos étnica, cultural y económicamente similares, lo que deriva en una alta homogenización interna (Alves, Elacqua, koslinki, Santos y Urbina, 2015).

Complementariamente, la presencia de una alta proporción de estudiantes con bajo nivel de logro concentrados en un mismo colegio limita, cuando no inhibe, la capacidad de la escuela para instalar valor agregado. Además, restringe las oportunidades que los estudiantes tienen de nutrirse positivamente de una influencia de pares que les permita progresar académicamente y establecer adecuados climas de convivencia. Evidencia disponible encuentra correlación entre niveles de rendimiento y climas áulicos, donde escuelas con deficientes resultados presentan también ambientes con relaciones deterioradas y conflictivas. Tanto la accesibilidad, permanencia, rendimiento educativo y convivencia de niños, niñas y adolescentes con ascendencia mapuche no está exenta de problemas ni manifestación de prejuicios (Figueroa Huencho, 2012), lo cual torna la labor pedagógica e inclusión de estos estudiantes un asunto complejo (González, 2017).

Los estudiantes mapuches asisten en mayor proporción a colegios con dependencia Municipal $(49,7 \%)$ y que atienden preferencialmente a grupos socioeconómicos Bajos y Medio bajos. Un 83,7\% se matricula en unidades educativas donde los padres y madres cuentan con reducidos capitales educativo y económico, características que según diversos hallazgos obstaculizan el adecuado desarrollo de trayectorias educativas (Cornejo y Redondo, 2007; Valenzuela, Bellei y De Los Ríos, 2010; Bellei, 2015; Sevilla, 2017). Investigaciones encuentran recurrente correlación entre el nivel de logro y el capital socioeconómico familiar, de modo que los alumnos que provienen de los sectores más vulnerables alcanzan peor desempeño escolar y asisten a establecimientos con baja calidad educativa (Monarca, 2012; Durán, 2018).

Los procesos educativos se ven afectados no solo por la calidad de los recursos escolares, las habilidades pedagógicas y el manejo disciplinar de los y las docentes, sino también por la disponibilidad de recursos materiales y simbólicos en el hogar, siendo particularmente relevante el capital educativo parental. A medida que el estudiante mapuche avanza curricularmente, el acompañamiento parental educativo decae, situación que intensifica el riesgo académico, segregación educativa y transmisión intergeneracional de carencias en el hogar.

Padres con mayor nivel de escolaridad tienden a otorgar mayor valor a la educación, invierten más en ella y presentan mejor predisposición al acompañamiento educativo (Rich y Jennings, 2015; Razeto, 2016; Sánchez, Reyes y Villarroel, 2016). Esta calidad educativa del hogar se precariza en contextos de pobreza, particularmente en familias mapuches que muestran un eventual desinterés cuando no resistencia al modelo educativo formal (Altschul, 2012; Valdés, Carlos y Arreola, 2013), agravado por políticas educativas que no toman en consideración la especificidad de la identidad étnica (Fernández y Fuentes, 2018).

\section{Conclusiones}

El Estado de Chile mantiene un conflicto abierto con el pueblo mapuche, por lo que su inclusión sigue siendo tarea pendiente. Si bien su exposición a procesos de transición demográfica ha significado modificar algunas condiciones y estilo de vida, ha mantenido su vinculación de precariedad y vulnerabilidad, especialmente en lo que a servicios sociales y educativos refiere. Por tal motivo, y en razón de la relevancia cultural que reviste, este artículo explora las condiciones de vida y patrones de carencialidad entre población mapuche, especialmente los ligados a la dimensión educativa. 
Este grupo étnico mayoritario reside principalmente en la macrozona sur del país y representa un $8,1 \%$ de la población nacional, establecida entre las regiones de Nuble y Los Lagos, y concentra los mayores índices de vulnerabilidad con independencia del enfoque de estimación de pobreza utilizado. Desde una perspectiva multidimensional, sus principales carencias refieren a componentes educativos, de accesibilidad y entorno, exhibiendo importantes brechas respecto de la población no mapuche en cada uno de los indicadores; situación transversal en la historia de las comunidades indígenas.

La pérdida de tierras y subdivisión predial, el empobrecimiento y erosión del suelo, sumado a la amenaza que supone la extensión de las plantaciones forestales, condiciona en estos grupos la adopción de una economía de subsistencia en territorios con escasa viabilidad agrícola. Junto con ser fuente de pobreza, este escenario fuerza a un permanente proceso de migración, particularmente acentuado entre jóvenes que buscan cumplir expectativas de educación y trabajo. De este conjunto de situaciones deriva que la identidad étnica no refiera exclusivamente a lo rural, y que tanto su estilo de vida y cosmovisión originaria estén en riesgo por procesos de aculturación.

En términos de inserción educativa, los jóvenes mapuches registran una mayor tendencia a matricularse en establecimientos de administración pública y que atienden preferencialmente a familias de menores recursos educativos y económicos. Los mecanismos de selección escolar y elección educativa parental han profundizado los procesos de segmentación socioeducativa, polarizando las adscripciones escolares de acuerdo a atributos de etnia.

Por otra parte, los hallazgos de este estudio nos permiten sostener que, no obstante, las mejoras en los índices de cobertura y retención, la calidad de la educación a la que tienen acceso los grupos mapuches continúa siendo deficiente. Si bien la incapacidad para instalar valor agregado es un atributo estructural del segregado sistema educativo chileno, está particularmente localizado entre establecimientos que atienden a población mapuche.

En efecto, los estudiantes mapuches no tan solo exhiben menores niveles de logro en pruebas estandarizadas respecto a la población no mapuche, sino que también asisten a lugares con alta prevalencia de alumnos/as con rendimiento insuficiente, es decir, comparten aulas compuestas significativamente por compañeros/as que no comprenden ni aplican los contenidos elementales del currículum en Matemáticas y Lenguaje. Empobrecido ambiente de aprendizaje que limita la capacidad de nutrirse cognitiva y actitudinalmente por la influencia de los pares.

La educación formal en Chile siempre ha sido un campo conflictivo dada su organización centralizada y un funcionamiento que segrega, y si bien se han incorporado políticas institucionales tendientes a una mayor inclusión, persisten prácticas de aislamiento y exclusión simbólica, particularmente cuando se trata de integración intercultural. En tal sentido, se hace necesario reforzar las medidas que reconozcan y favorezcan la pluriculturalidad como enriquecedora del proceso educativo.

\section{Referencias}

Agencia de Calidad de la Educación (2018). Resultados Evaluación de calidad educativa 2018 [Fichero de datos]. Recuperado de https:/www. agenciaeducacion.cl/

Becerra, S., Beldaño, C., Dastro, A., \& Coñuepan, J. (2011). Prejuicio y discriminación étnica docente hacia niños indígenas en la escuela. Teoria e Prática da Educação, 14(1), 7-17.

Durán Sanhueza, F. (2018). La Evaluación de la calidad educativa en Chile: instrumentos de control y rendición de cuentas. Revista Educación, Política y Sociedad, 3(1), 85-99.

Encuesta de Caracterización Socioeconómica Nacional (2017). Resultados CASEN 2017 [Fichero de datos]. Recuperado de http://observatorio. ministeriodesarrollosocial.gob.cl/casen-multidimensional/

casen/casen_2017.php

Quintriqueo , S., \& Torres , H. (2012). Distancia entre el conocimiento mapuche y el conocimiento escolar en contexto mapuche. Revista Electrónica de Investigación Educativa, 14(1), 16-33.

Sánchez , A., Reyes, F., \& Villarroel, V. (2016). Participación y expectativas de los padres sobre la educación de sus hijos en una escuela pública. Estudios pedagógicos (Valdivia), 42(3), 347-367.

Segato, R. (2012). Racismo, Discriminación y Acciones Afirmativas: Herramientas Conceptuales. Observatório da Jurisdição Constitucional, 1(1), $1-16$.

Altschul, I. (2012). Linking socioeconomic status to the academic achievement of mexican american youth through parent involvement in education. Journal of the Society for Social Work and Research, 3(1), 13-30. doi:10.5243/jsswr.2012.2

Alves, F., Elacqua, G., koslinki, M., Santos, H., \& Urbina, D. (2015). Ganadores y perdedores de la elección de la escuela: evidencia de Río de Janeiro, Brasil y Santiago, Chile. Internacional de Desarrollo Educativo, 41, 25-34.

Arias, K., \& Bravo, P. (2019). (Des) encuentros en la Educación Intercultural en Contexto Mapuche, Chile. REXE-Revista de Estudios y Experiencias en Educación, 18(36), 177-191.

Bellei, C. (2015). El gran experimento: Mercado y privatización de la educación chilena. Santiago: LOM. 
Bolados, P. (2012). Neoliberalismo multicultural en el Chile postdictadura: la política indígena en salud y sus efectos en comunidades mapuches y atacameñas. Chungará (Arica), 44(1), 135-144.

Cornejo, R., \& Redondo, J. (2007). Variables y factores asociados al aprendizaje escolar. Una discusión desde la investigación actual. Estudios Pedagógicos, 33(2), 155-175.

Correa, N. (2018). Iniciativas públicas y privadas a favor de la pobreza rural indígena y afrodescendiente en América Latina: balance y perspectivas. Lima: Instituto de Estudios Peruano.

Cunningham, M. (2010). La situación de los pueblos indígenas del mundo. Nueva York: Departamento de Información Pública de las Naciones Unidas.

Díaz Fuentes, R., Osses Bustingorry, S., \& Muñoz Navarro, S. (2016). Factores e interacciones del proceso de enseñanza-aprendizaje en contextos rurales de la Araucanía, Chile. Estudios pedagógicos (Valdivia), 42(3), 111-128.

Espinoza, C. (2018). El desafío municipal mapuche. Etnografía de una experiencia política: Tirúa 1992-2008. Revista de Antropología Iberoamericana, 13(3), 355 - 379.

Espinoza, Ó., Castillo, D., González, L., \& Loyola, J. (2014). Factores familiares asociados a la deserción escolar en los niños y niñas mapuche: un estudio de caso. Estudios pedagógicos (Valdivia), 40(1), 97-112.

Fernández, J., \& Fuentes, C. (2018). Percepciones sociales sobre el derecho de autonomía de los pueblos indígenas en Chile. Polis (Santiago), 17(49), 29-54. doi:https:// dx.doi.org/10.4067/S0718-65682018000100029

Fernández, M., \& Hauri, S. (2016). Resultados de aprendizaje en la Araucanía. La brecha de género en el SIMCE y el androcentrismo en el discurso en el discurso de docentes de Lenguaje y Matemáticas. Calidad en la Educación(45), 54-89.

Figueroa Huencho, V. (2012). La realidad de los pueblos indígenas en Chile: una aproximación sociodemográfica para contribuir al diseño de políticas públicas pertinentes. Anales de la Universidad de Chile(3), 137-153. doi:10.5354/0717-8883.2012.21733

Gallart, M., \& Henríquez, C. (2006). Indígenas y educación superior: algunas reflexiones. Universidades(32), 27-37.

González, R. (2017). Proyecto amistad: relaciones entre niños indígenas y no indígenas en las escuelas. Santiago de Chile: CIIR.

Henry, M. (2010). Working with/against globalization in education. Journal of Educational Policy, 14(1), 85-97.

Hopenhayn, M., \& Bello, M. (2001). Discriminación étnico-racial y xenofobia en América Latina y el Caribe. Cepal.

Infante, M. (2010). Desafíos a la formación docente: inclusión educativa. Estudios pedagógicos (Valdivia), 36(1), 287-297.

Juárez-Ramírez, C., Márquez-Serrano, M., Salgado de Snyder, N., Pelcastre-Villafuerte, B., Ruelas-González, M., \& Reyes- Morales, H. (2014). La desigualdad en salud de grupos vulnerables de México: adultos mayores, indígenas y migrantes. Revista Panamericana de Salud Pública, 35, 284-290.

Mendoza, R. (2017). Inclusión educativa por interculturalidad: implicaciones para la educación de la niñez indígena. Perfiles educativos, 39(158), 52-69.

Mickelson, R., Bottia, M., \& Lambert, R. (2013). Effects of School Racial Composition on K-12 Mathematics Outcomes: A Metaregression Analysis. Review of Educational Research, 83(1), 121-158.

Miller,A. (2012). La Falta de Desarrollo Debido a la Carencia de Igualdad: La Ineficacia del Programa de Educación Intercultural Bilingüe Como Factor de Continuación de la Dominación del Estado de Chile Contra el Pueblo Mapuche. Independent Study Project (ISP) Collection

Monarca, H. (2012). La racionalidad de las políticas de evaluación de la calidad de la educación. Revista Iberoamericana de educación, 59(1), 1-9.

Montero, R., \& Garcés, P. (2009). ¿Existe discriminación salarial contra la población indígena en Chile? El Trimestre Económico, 645-669.
Moreno-Doña, A., \& Gamboa, R. (2014). Dictadura chilena y sistema escolar: "A otros dieron de verdad esa cosa llamada educación". Educar em Revista, 51, 51-66. doi:10.1590/ S0104-40602014000100005

Noe, D., Rodríguez, J., \& Zuñiga, I. (2005). Brecha étnica e influencia de los pares en el rendimiento escolar: evidencia para Chile. Vol. 102. Santiago de Chile: United Nations Publications.

Ortega, M. (2019). Correlación entre la inclusión y el SIMCE. Revista Scientific, 4, $18-37$.

Peña, C. (2007). Por qué no debemos seleccionar. En J. J. Brunner, \& C. Peña (Edits.), La reforma al sistema escolar: aportes para el debate (págs. 245-252). Santiago de Chile: Universidad Diego Portales .

Quintrileo, C., Yáñez, C., \& Valenzuela, C. (2013). Una aproximación crítica a la propuesta en consulta del Programa de Educación Intercultural Bilingüe (PEIB) en Chile. Revista de Lingüística, Filosofía y Literatura, 23(1), 45-61.

Raguileo, M., \& Escobar, J. (2016). Una aproximación a la vulnerabilidad en el sistema de educación parvularia en Chile. Nóesis. Revista de Ciencias Sociales y Humanidades, 25(50), 51-88.

Rapimán, D. (2005). Educación intercultural desde la teoría del control cultural en contexto de diversidad sociocultural mapuche. Cuadernos Interculturales, 3(4), 37-50.

Razeto, A. (2016). El involucramiento de las familias en la educación de los niños. Cuatro reflexiones para fortalecer la relación entre familias y escuelas. Páginas de Educación, 9(2), 190-216. doi:10.22235/pe.v9i2.1298

Rich, P., \& Jennings, J. (2015). Choice, information, and constrained options: School transfers in a stratified educational system. American Sociological Review, 80(5), 10691098 .

Sanchez, A. (2011). Etnia y rendimiento académico en Colombia. Revista de Economía del Rosario, 14(2), 189-227.

Serrano, S., \& Ponce de León, M. (2018). Historia de la educación en Chile (18102010). 3 .

Sevilla, P. (2017). Diferenciación Curricular y Estratificación Escolar: El impacto de la polivalencia en la educación media chilena. Santiago de Chile: Universidad Alberto Hurtado - Universidad Diego Portales.

Stillerman, J. (2016). Educar a niñas y niños de clase media en Santiago: capital cultural y segregación socioterritorial en la formación de mercados locales de educación. EURE (Santiago), 42(126), 169-186. doi:10.4067/S0250-71612016000200008

Trivelli, C. (2008). Persistente desigualdad entre indígenas y no indígenas en América Latina. Documento de trabajo/Programa Dinámicas Territoriales Rurales. RIMISP-Centro Latinoamericano para el Desarrollo Rural.

Turra-Díaz, O. (2012). Currículo y construcción de identidad en contextos. Educación y Educadores, 15(1), 81-95.

UNESCO. (2009). Directrices sobre políticas de inclusión en la educación. París: UNESCO

Vain, P. (2009). Escuela, Estado y familia. Un pacto por redefinir. Educación, lenguaje y sociedad, 6(6), 329-344.

Valdés, A., Carlos, E., \& Arreola, C. (2013). Desarrollo de un instrumento para medir la participación de los padres en la educación escolar de los hijos. Revista de Evaluación Educativa, 2(1), 92-109.

Valenzuela, J. P., Bellei, C., \& De Los Ríos, D. (2010). Segregación escolar en Chile. En S. Martinic, \& G. Elacqua (Edits.), Cambios en la gobernanza del sistema educativo chileno (págs. 257-284). Santiago de Chile: UNESCO, Pontificia Universidad Católica de Chile.

Vejar, D., Marchant, F., \& Peña, E. (2018). Documento de trabajo: caracterización de trabajadoras mapuche en la región de la Arancanía. Temuco: Grupo de estudios del trabajo desde el sur.

Webb , A., Canales, A., \& Becerra, R. (2017). Las desigualdades invisibilizadas: población indígena y segregación. 